\title{
The relevance of a digital platform for the citizen science project NestCams
}

\author{
Julia Rittenschober ${ }^{a,}$, Verena Puehringer-Sturmayr ${ }^{a, b}$ and Didone Frigerio ${ }^{a, b}$ \\ a Konrad Lorenz Research Center, Core Facility for Behavior and Cognition, University of Vienna, \\ Fischerau 11, 4645 Gruenau im Almtal, Austria \\ $b$ Department of Behavioral and Cognitive Biology, University of Vienna, \\ Althanstrasse 14, 1090 Vienna, Austria \\ E-mail: julia.rittenschober@univie.ac.at,verena.puehringer-sturmayr@univie.ac.at, \\ didone.frigerio@univie.ac.at
}

Digital platforms create opportunity for citizen scientists to become involved in the analysis of large data sets. In the present study, we discuss the relevance of the platform 'Zooniverse' and the reliability of citizen scientists with regard to data analysis, which we explore using NestCams as a case study. 


\section{Introduction}

Citizen science is a research format that involves volunteers in scientific research, thereby generating new knowledge and providing benefits for both the participants and the scientists [1]. Since the mid-2000s, an increasing number of projects have been using digital platforms to analyse large datasets including, for example, pictures and videos [1]. This approach offers a broad range of reach and fosters visibility for the research group. The online platform 'Zooniverse' (https://www.zooniverse.org) hosts numerous citizen science projects worldwide showcasing cutting edge research from different scientific disciplines (e.g. biology, climate change, arts, history or literature) [2]. Additionally, digital platforms consider the reliability of the observers and the possibility of several volunteers working in parallel on the same dataset [1].

In the present study, we use the project 'NestCams' as a case study and examine the relevance of the digital platform 'Zooniverse' and the reliability of citizen scientists. The aim of 'NestCams' is to identify which behaviours influence the hatching success of eggs in two avian species, the Greylag Goose (Anser anser) and the Northern Bald Ibis (Geronticus eremita), with observations that hatching success may range from $0 \%$ to $100 \%$. In this analysis, we focus on the Greylag Goose.

We address the following questions: (1) what is the relevance of a digital platform like 'Zooniverse' for data analysis for our project NestCams? (2) How reliable are the obtained data?

\section{Methods \& Results}

During two breeding seasons, motion-sensitive cameras (RPI CAM 5MP, sensor CMOS1/4) were installed at 16 Greylag Goose breeding huts and recorded short video sequences of the activities inside the nest. In total, more than 100,000 hours of video footage were recorded. Because of the vast amount of recordings, we decided to enlist volunteers for data extraction via the platform 'Zooniverse'. 'Zooniverse' was selected for our citizen science project, as it is the online platform with the widest reach in terms of citizen science projects. The videos were randomly allocated to video-packages of 500 recordings and uploaded on the online platform. Citizen scientists watch and analyse the videos by answering two questions and using provided answers: (1) Is the Greylag Goose sitting or standing on the nest? (i.e. incubating or not), and (2) Which behaviour is the Greylag Goose showing on the nest? (i.e. coming back to the nest, leaving the nest, egg rolling, parenting).

Participants are introduced to the tasks through a 'tutorial' (i.e. pictures and step-by-step introduction of the project) and by a 'field guide' (i.e. descriptions and videos for each behaviour). Additionally, a 'chat' section (so called 'Talk') is available for the communication between scientists and volunteers, who can share videos they are unsure about and get an expert opinion on it. About 1,000 questions and comments were posted from March 2019 to August 2020. Though the flow of such questions is rather irregular, a quick response is generally welcome.

NestCams has been available online since spring 2019. By the end of August 2020, 3,949 users had performed 80,807 video codings.

Third Mission activities were used to advertise the project and recruit volunteers. At the Children University offered in Upper Austria, about 140 children and young people aged 9 to 15 years had the opportunity to analyse NestCams videos and coded more than 1,200 videos. 
NestCams was also part of the Citizen Science Award (a competition for citizen scientists in Austria with a focus on schools).

The monthly classifications illustrate the importance of digital platforms and the large network of citizen scientists (Figure 1). In 2019, 2,878 \pm 729 videos (mean \pm SE) were coded on average per month, while we registered $6,398 \pm 1,445$ video codings (mean \pm SE) on average per month in 2020. Right after the official project launch on Zooniverse (September 2019) and during the 2020 COVID-19 pandemic and lockdown (from March onwards), we noted a spike in video codings.

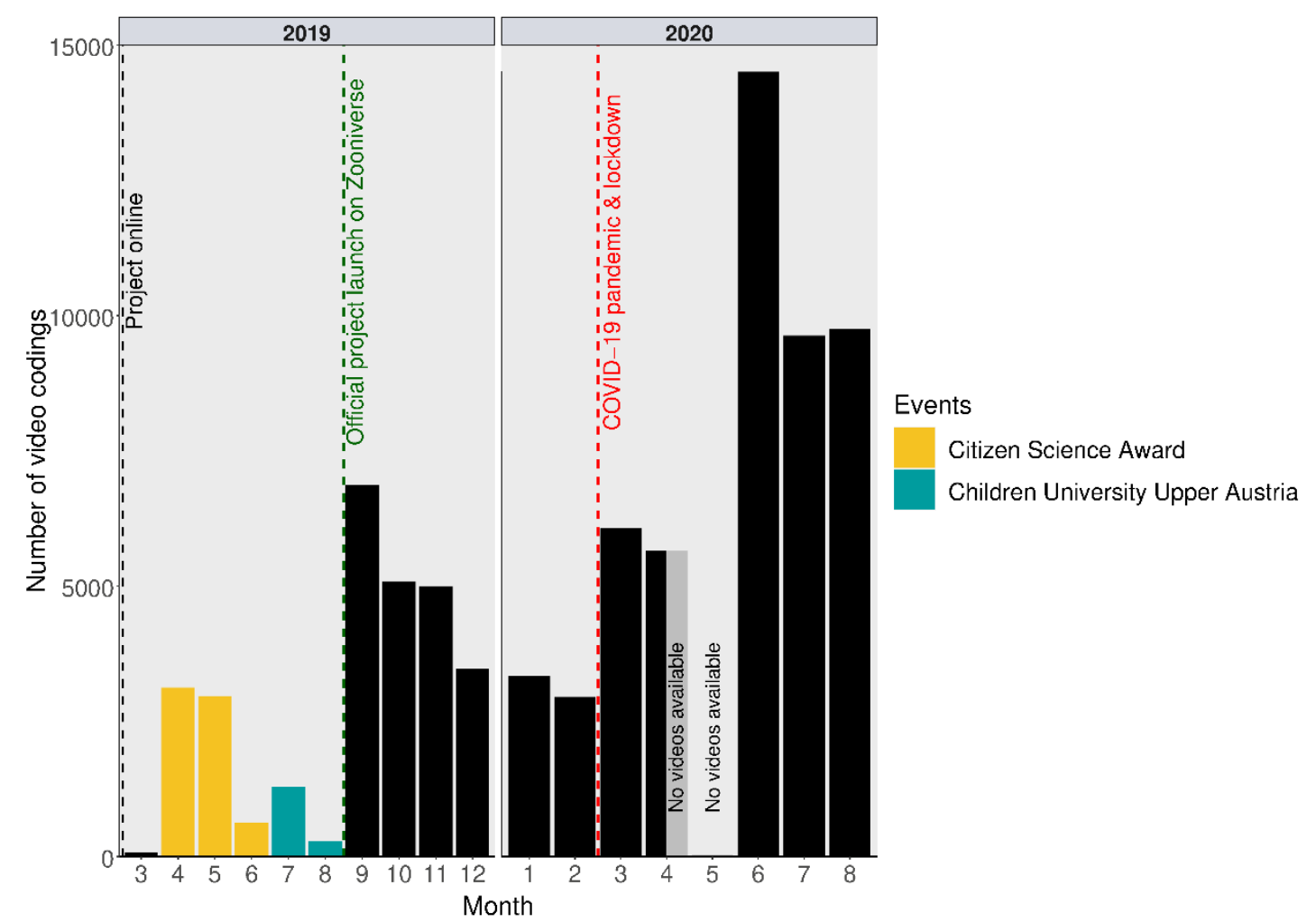

Figure 1. Local (i.e. Citizen Science Award, Children University in Upper Austria) and worldwide content coding for the project 'NestCams' on the online platform 'Zooniverse'. Dashed lines indicate the month in which the project went online (black dashed line), the official project launch on Zooniverse (green dashed line), and the start of the COVID-19 pandemic and lockdown in Austria (red dashed line). Events at which the project NestCams was applied are marked in colour.

The reliability of the citizen scientists was calculated by analysing more than 2,500 videos by experts (i.e. members of the research team and NestCams experienced users) and comparing those to the same videos analysed by citizen scientists (i.e. NestCams users and participants of the Children University). The reliability of NestCams users and participants of the Children University was high in both questions (Figure 2). 


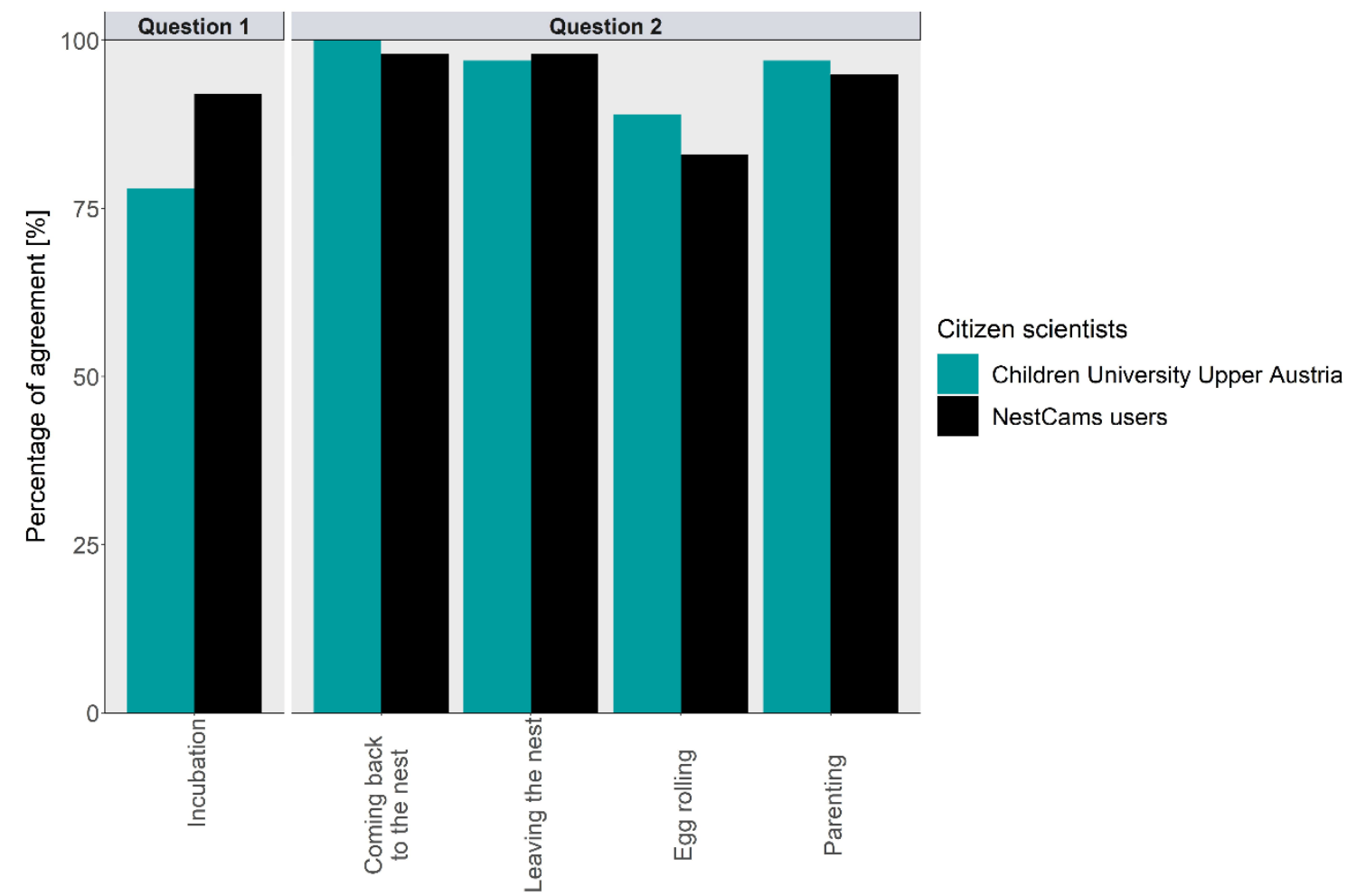

Figure 2. Reliability of data between experts and citizen scientists (i.e. participants of the Children University and NestCams users). Participants of the Children University are coloured in blue, NestCams users in black. Reliability was calculated for Question 1 (Is the Greylag Goose sitting or standing on the nest? This corresponds to incubation) and Question 2 (Which behaviours is the Greylag Goose showing on the nest?).

\section{Discussion}

The digital platform 'Zooniverse' was useful for data analysis for our project 'NestCams', where large datasets need to be analysed in a reasonable time. The reliability of the users was increased through tutorials, the field guide, and online chat sessions that provided sufficient background information to successfully code the videos.

'NestCams' benefits from the wide network of volunteers on Zooniverse, with a sharp increase in the number of codings after the official launch on Zooniverse (Figure 1). The local, national and international scope of a citizen science project can have an immense influence on the applicability and extent of data acquisition and analysis. The influence of the COVID-19 pandemic had a measurable impact on amount of coding. However, the biggest constraints for participation seem to be time and personal reasons, as stated in an evaluation of the Planet Hunters project on Zooniverse [3].

The reliability of the data between citizen scientists and experts was good. However, about $10 \%$ of the videos were not clearly answered by different citizen scientists and/or experts. Low reliability might be additionally affected by the quality of the video itself (i.e. the nest may not have been optimally captured on camera) or interruption of the video sequence related to technical settings. Possibly not all citizen scientists used the tutorial and step-by-step introduction. The 'chat' section, for instance, is only actively used by about $5 \%$ of the users. 
Good communication between researchers and citizen scientists as well as great support and guidance through the project are crucial and necessary for high quality data acquisition. Apart from requesting clear explanations and instructions, participants, for example, ask for individual expert opinions on the videos; in addition, they also give feedback on the project in general and the project design and ask questions about the research topic or the study species in the 'chat' section. However, as only about $5 \%$ of the users actively use the 'chat' section, communication between researchers and citizen scientists could be expanded through other channels, e.g. social media, eNewsletters, blog posts, or WhatsApp could serve as a direct communication channel. Thus, it is important not to underestimate the value and effort required for communication.

Our findings suggest that digital platforms are of great relevance for citizen science projects, not least because of the international network, the possibility to manage and analyse large datasets within a shorter time frame and the high quality data acquisition.

\section{Acknowledgements}

We gratefully acknowledge K. Buchegger, V. Föger, E. Ganzenbacher, G. Gegendorfer, F. Hemetsberger, J. Hemetsberger, M. Hemetsberger, F. Hirschenhauser, E.-M. Körmer, L. Iaiza, M. Moser, Z. Simmer, K. Stadler, F. Steinmaurer, E. Stoik, L. Schwaiger, H. Vesely, M. Weidinger and E. Zorn for their valuable support in the different phases of the project. The cameras and the platform were implemented with the support of the companies Bogner\&Lehner Metereologie and Spotteron. The project was funded by the Austrian Research Promoting Agency (FFG, project number 858551). Permanent support came from the 'Herzog vom Cumberland Stiftung' and the 'Verein der Förderer der Konrad Lorenz Forschungsstelle'.

\section{References}

[1] Kullenberg, C., Kasperowski, D. (2016). What Is Citizen Science? - A Scientometric Meta-Analysis. PloS ONE, 11(1): e0147152

[2] Simpson, R., Page, K. R., De Roure, D. (2014). Zooniverse: observing the world's largest citizen science platform. Proceedings of the 23rd International Conference on World Wide Web, WWW' 14 Companion (New York: ACM), pp. 1049-1054

[3] Depper, A. (2019). Planet Hunters, Zooniverse Evaluation report. Oxford: University of Oxford, p. 24 